\title{
Aplicação de Modelos de Redes Neurais Artificiais na Previsão do Preço do Alumínio
}

\begin{abstract}
José Airton Azevedo dos Santos
Doutorado em Engenharia Elétrica pela Universidade Federal de Santa Catarina -

UFSC

Professor na Universidade Tecnológica Federal do Paraná - UTFPR Av. Brasil, 4232. Independência. Medianeira/PR. CEP: 85884-000

E-mail: airton@utfpr.edu.br

Yasmin Chaucoski Graduação em andamento em Engenharia Elétrica pela Universidade Tecnológica Federal do Paraná - UTFPR Av. Brasil, 4232. Independência. Medianeira/PR. CEP: 85884-000 E-mail: chaucoski@alunos.utfpr.edu.br
\end{abstract}

\section{RESUMO}

O alumínio é um metal não ferroso muito utilizado nas indústrias metalúrgicas, farmacêuticas, aeronáuticas e alimentares. O preço do alumínio apresenta muitos fatores de incerteza. Desse modo a sua previsão é muito importante na definição de políticas industriais, bem como para os produtores e consumidores. Neste contexto, este trabalho tem como objetivo avaliar a eficácia de modelos das redes neurais artificiais (RNAs) para a previsão do preço do alumínio. A base de dados apresenta uma série histórica do preço do alumínio no período entre 2006 e 2020. Modelos de previsão, baseados em Redes Neurais LSTM (Long Short-Term Memory) e MLP (Multilayer Perception) foram implementados na linguagem Python, utilizando o framework Keras. Resultados obtidos dos dois modelos que foram comparados. Verificou-se, também, para um horizonte de seis meses que o modelo LSTM apresentou um melhor desempenho que o modelo MLP.

Palavras-chave: MLP. LSTM. Alumínio.

\section{Application of Artificial Neural Network Models in Aluminum Price Forecast}

\section{ABSTRACT}

Aluminum is a non-ferrous metal, widely used in the metallurgical, pharmaceutical, aeronautical and food industries. The price of aluminum has many factors of uncertainty. Therefore, its forecast is very important in the definition of industrial policies, as well as for producers and consumers. In this context, the present work aims to evaluate the effectiveness of models of artificial neural networks (ANNs, for forecasting the price of 
aluminum. The database presents a historical series, of the price of aluminum, in the period between 2005 and 2020. Forecasting models, based on LSTM (Long Short-Term Memory) and MLP (Multilayer Perception) Neural Networks, were implemented in Python language using Keras framework. Results obtained from the two models were compared. We found for a six-month horizon, that the LSTM model performed better than the MLP model.

Keywords: MLP. LSTM. Aluminun.

\section{Aplicación de Modelos de Redes Neuronales Artificiales na Predicción del Precio del Aluminio}

\section{RESUMEN}

El aluminio es un metal no ferroso muy utilizado en las industrias metalúrgicas, farmacéuticas, aeronáuticas y alimentarias. El precio del aluminio presenta muchos factores de incertidumbre. Por tanto, su previsión es muy importante en la definición de políticas industriales, así como para productores y consumidores. En este contexto, este trabajo tiene como objetivo evaluar la efectividad de modelos de redes neuronales artificiales (ANN) para predecir el precio del aluminio. La base de datos presenta una serie histórica de precios del aluminio para el período comprendido entre 2006 y 2020. Los modelos de pronóstico, basados en redes neuronales LSTM (Long Short-Term Memory) y MLP (Multilayer Perception), se implementaron en lenguaje Python, utilizando el framework Keras. Se compararon los resultados obtenidos de los dos modelos. Se verificó, también para un horizonte de seis meses, que el modelo LSTM presentó un mejor desempeño que el modelo MLP.

Palabras clave: MLP. LSTM. Aluminio.

\section{INTRODUÇÃO}

O alumínio, o mais abundante entre os metais, é muito utilizado pela sociedade moderna. É derivado da bauxita, com um estágio intermediário da alumina, que é transformada em alumínio por eletrólise. Suas propriedades (leveza, alta condutibilidade elétrica, entre outras) permitem que sejam utilizados na produção de vários itens (Castro, 2015). 
O alumínio é utilizado na fabricação de veículos (indústria automotiva); em embalagens do setor farmacêutico, de bebidas, etc. (indústria de embalagens); em linhas de transmissão de energia elétrica (indústria de energia) e em bens de consumo está presente em assadeiras, panelas, entre outros (Cardoso, 2011).

Segundo Castro (2015), a possibilidade do preço do alumínio ser frequentemente aleatório traz incertezas no processo de análise do mercado, tornando a previsão do preço do alumínio de extrema relevância, dado seu impacto na cadeia industrial. As previsões do preço do alumínio podem fornecer importantes informações e subsídios para o processo de tomada de decisão.

Redes neurais artificiais são abstrações da rede neural biológica. São capazes de memorizar, analisar e processar um grande número de dados obtidos de um experimento. Seu objetivo não é replicar, mas sim servir de modelo para o aprendizado e resoluções de problemas complexos (Sebastian, 2016; Pinheiro, Santos, \& Pasa, 2020; Bastiani, Santos, Schmidt, \& Sepulveda, 2018).

Neste contexto, este trabalho apresenta uma aplicação de modelos de Redes Neurais Artificiais (RNAs) para previsão do preço do alumínio, no período de 1 de janeiro de 2006 a 30 de junho de 2020.

As RNAs têm sido utilizadas com sucesso em tarefas de predição e modelagem de séries temporais (Haykin, 2001). Segundo Moore, Mccabe, Duckworth e Sclove (2006), séries temporais são medidas de determinadas variáveis (preço, produção etc.) tomadas em intervalos regulares de tempo. Um modelo de séries temporais fornece previsões de observações futuras por meio da relação que possui com valores passados (Morettin \& Toloi, 2004).

\section{REFERENCIAL TEÓRICO}

\subsection{Trabalhos Relacionados}

O tema, previsão do preço do alumínio, vem sendo abordado em diversos trabalhos ao longo dos anos. Dentre eles pode-se citar os trabalhos de Castro (2015) 
que utilizou, nas previsões do preço do alumínio, os modelos ARIMA E ARIMAX. Utilizou, neste trabalho, uma base de dados de janeiro de 1999 a setembro de 2014. Ru e Ren (2012) propuseram um método ARMA para previsão do preço do alumínio. Observaram, em suas análises, que o modelo ARMA se ajusta muito bem as flutuações do preço do metal. Kriechbaumer, Angus, Parsons e Casado (2014) avaliaram a utilização de uma estrutura Wavelet-ARIMA na previsão dos preços mensais do alumínio. Determinaram, por meio deste estudo, que a estrutura proposta aumentou a precisão das previsões. Castro et al. (2013), compararam a capacidade de previsão de modelos, VAR, VEC, estrutural e ARIMA, com base nos preços spot do alumínio. Relataram que o modelo VEC apresentou melhor desempenho de previsão.

\subsection{Modelos Quantitativos e Qualitativos}

Os modelos de previsão, quanto ao tipo de abordagem, podem ser classificados como qualitativos ou quantitativos. Os modelos qualitativos possuem caráter subjetivo e utilizam em suas análises fatores como experiência profissional ou intuição. Já os quantitativos, utilizados neste trabalho, caracterizam-se por utilizar ferramentas matemáticas. São divididos em intrínsecos (Séries Temporais) e extrínsecos (Regressões) (Tubino, 2009).

\subsection{Redes MLP}

As redes MLPs representam uma classe de redes neurais utilizadas na modelagem de sistemas complexos. Sendo muito utilizadas na previsão de dados financeiros porque podem aproximar muito bem funções não lineares. As redes MLPs são compostas por uma camada de entrada, uma ou mais camadas ocultas de unidades de processamento e uma camada de saída. Seu treinamento é supervisionado e utiliza o algoritmo backpropagation. O backpropagation é o algoritmo responsável pelo aprendizado da rede. As redes MLPs podem realizar tanto regressão quanto classificação (Spancerski \& Santos, 2021; Pinheiro et al., 2020; Bastiani et al., 2018). 


\subsection{Redes LSTM}

A rede LSTM é uma Rede Neural Recorrente (RNR) projetada para modelar sequências temporais e suas dependências de longo prazo, com maior precisão que as redes neurais recorrentes convencionais. Atualmente, esse tipo de rede é muito utilizada porque opera com boa precisão em uma grande variedade de problemas (Zao, 2017).

A topologia, de um neurônio, de uma LSTM é baseada em uma célula de memória. Uma célula de memória possui um componente de memória para armazenar sequências recentes, o que o torna mais inteligente que um neurônio clássico. Cada célula contém portas, as quais controlam a quantidade de informação que é memorizada e a quantidade de informação que deve ser repassada para as próximas unidades (Nelson, Pereira, \& Oliveira, 2017).

Um neurônio LSTM (célula) trabalha com uma sequência de entrada $x_{t}$ (Figura 1) e cada porta (gate), dentro de uma célula, usa unidades de ativação para controlar se elas são acionadas ou não, fazendo com que a mudança de estado e a adição de informações fluam através da célula. $O$ parâmetro $C_{t}$ representa o estado da célula no instante $t$, esse estado representa as informações que chegaram até esse passo em instante de tempos passados. O gate de esquecimento $f_{t}$ determina quais informações devem ser jogadas fora pela célula. O gate de entrada $i_{t}$ determina os valores de entrada para atualizar o estado da célula e o gate de saída $O_{t}$ determina o que produzir com base na entrada e na memória da célula. Todos esses valores acabam sendo concatenados, multiplicados ou somados, conforme mostra o circuito apresentado na Figura 1 (Graves, 2014). 


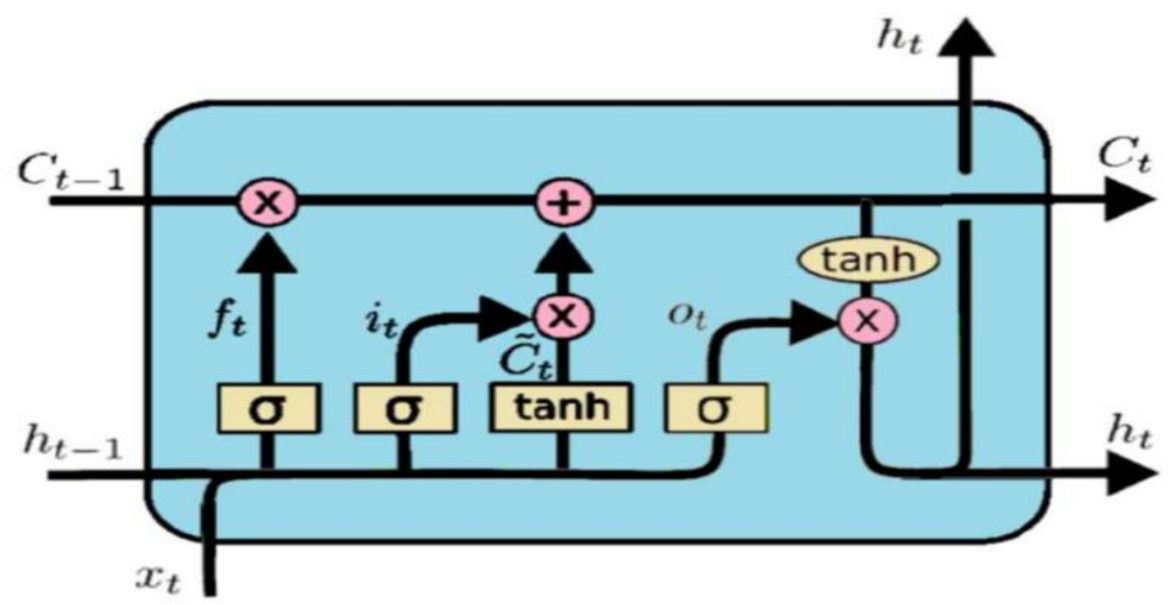

Figura 1. Célula LSTM

Fonte: Graves (2014).

\section{PROCEDIMENTOS METODOLÓGICOS}

Inicialmente, neste trabalho, realizou-se a análise exploratória dos dados obtidos do Word Bank (IndexMundi, 2020), com a intenção de obter informações importantes sobre os mesmos. Em seguida, realizou-se a etapa de modelagem, em que foram implementados vários modelos de redes neurais MLP e LSTM. Nesta etapa os modelos com melhor desempenho, no conjunto de teste, foram selecionados. Observa-se que os modelos de séries temporais, do preço do alumínio, incluem uma camada de entrada, uma camada oculta e uma camada de saída.

Finalmente, na última etapa, fez-se a validação dos modelos das RNAs, analisando-se a acurácia, dos modelos, com dados que não participaram do processo de treinamento e teste.

\subsection{Bases de Dados}

Para previsão, do preço do alumínio, utilizou-se uma base de dados com 174 meses (Jan/2006 - Jun/2020) obtida do Word Bank (Aluminum, 99.5\% minimum purity, 
LME spot price, CIF UK ports) (IndexMundi, 2020). Os dados obtidos, da base de dados, já estavam limpos e sem a presença de outliers. Na Figura 2 apresenta-se o boxplot dos dados.

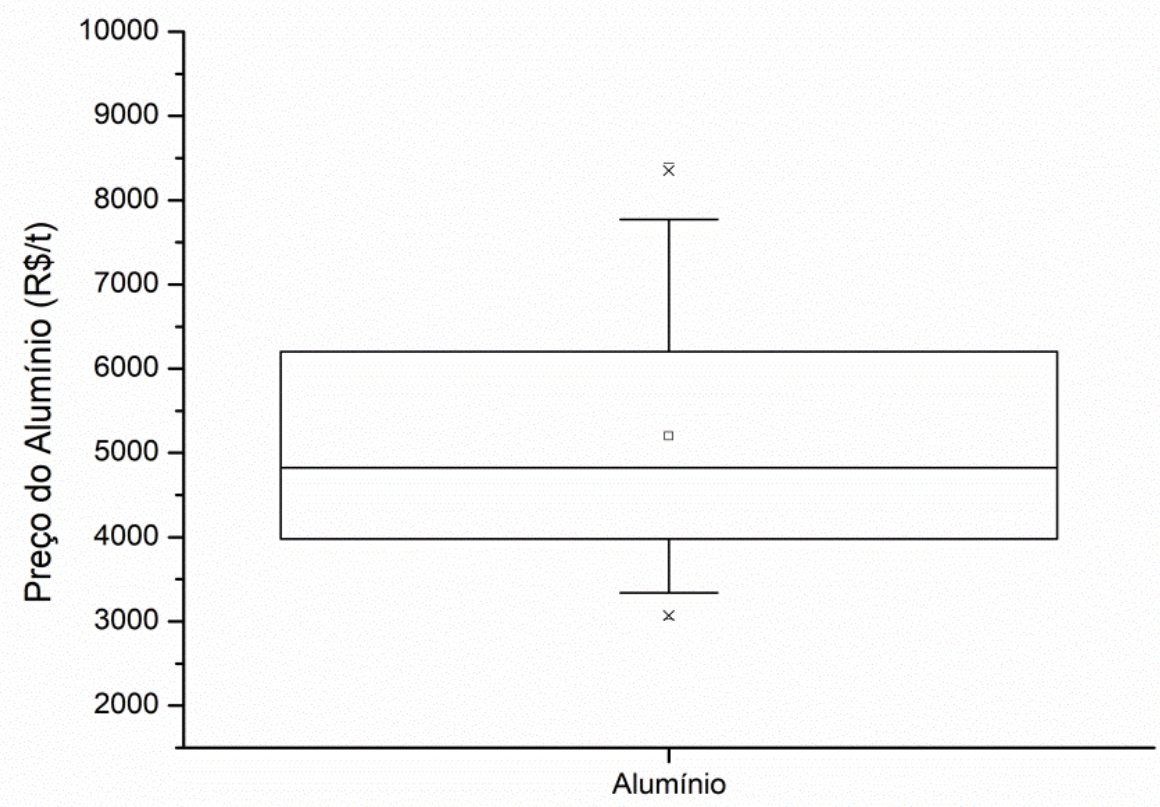

Figura 2. Boxplot

Fonte: Autoria própria (2020). 
Os dez primeiros registros do conjunto de dados são apresentados na Tabela 1.

Tabela 1

Dez primeiros registros do arquivo de dados

\begin{tabular}{cc}
\hline Ano & Alumínio $(\mathbf{R} \mathbf{\$} / \mathbf{t}))$ \\
\hline $2006-1$ & 5931,08 \\
$2006-2$ & 5774,89 \\
$2006-3$ & 5720,65 \\
$2006-4$ & 5668,03 \\
$2006-5$ & 5144,56 \\
$2006-6$ & 5146,73 \\
$2006-7$ & 4920,09 \\
$2006-8$ & 4537,27 \\
$2006-9$ & 4409,02 \\
$2006-10$ & 4437,43 \\
\hline
\end{tabular}

Nota. Fonte: IndexMundi (2020).

\subsection{Método de Fragmentação e Critério de Parada}

Para criar os subconjuntos de dados, de treinamento e teste foram usados 168 observações da base de dados do Word Bank (IndexMundi, 2020). Observa-se que os preços relativos aos meses de janeiro, fevereiro, março, abril, maio e junho de 2020 foram retirados do conjunto de dados para serem utilizados posteriormente para validação dos modelos. Neste trabalho, utilizou-se o método de fragmentação de Houldout onde a base de dados foi dividida com $67 \%$ dos dados para treinamento dos algoritmos e $33 \%$ para teste.

Como critério de parada utilizou-se o método conhecido como Método de Parada Antecipada (Earling Stopping). Segundo Silva (2010), esse método ajuda a projetar uma rede neural com bom poder de generalização. Neste contexto, definiu-se como critério de parada do treinamento, a função EarlyStopping() com o parâmetro patience $=50$. $O$ parâmetro patience indica o número de épocas, após a qual nenhuma melhoria foi observada. 


\subsection{Métricas}

Neste trabalho, os modelos obtidos foram avaliados pelas métricas apresentadas no Quadro 1 (Cankurt \& Subasi, 2015).

\begin{tabular}{|c|}
\hline Coeficiente de Determinação $\left(\mathbf{r}^{2}\right)$ \\
\hline $\begin{array}{c}\text { A qualidade de ajuste de um modelo pode ser avaliada pelo coeficiente de determinação. Este } \\
\text { coeficiente indica quanto o modelo foi capaz de explicar os dados coletados. }\end{array}$ \\
\hline Raiz Quadrada do Erro Médio Quadrático (RMSE) \\
Raiz do erro médio quadrático da diferença entre a predição e o valor real. Tem sempre valor positivo e \\
quanto mais próximo de zero, maior a qualidade dos valores preditos.
\end{tabular}

Quadro 1. Métricas

Fonte: Cankurt e Subasi (2015).

\section{RESULTADOS E DISCUSSÃO}

Inicialmente, neste trabalho, realizou-se uma análise descritiva dos dados (Tabela 2).

Tabela 2

Análise descritiva dos dados

\begin{tabular}{l|c}
\hline Média $(\mathrm{R} \$ / \mathrm{t})$ & 5198,94 \\
\hline Mínimo $(\mathrm{R} \$ / \mathrm{t})$ & 3027 \\
\hline Máximo $(\mathrm{R} \$ / \mathrm{t})$ & 8435,55 \\
\hline Desvio Padrão $(\mathrm{R} \$ / \mathrm{t}))$ & 1450,52 \\
\hline Coeficiente de Variação (\%) & $27,9 \%$ \\
\hline
\end{tabular}

Nota. Fonte: Dados da pesquisa (2020). 
Pode-se observar nos dados apresentados na Tabela 2, que o preço, para o período em estudo, ficou em média 5198,94 R\$/t., apresentando, neste período, preços mínimo e máximo de $3027 \mathrm{R} \$ / \mathrm{t}$ e $8435,55 \mathrm{R} \$ / \mathrm{t}$, respectivamente.

Observa-se também, na Tabela 2, que o coeficiente de variação $(27,9 \%)$ é considerado alto, o que indica variabilidade dos dados.

A série histórica do preço do alumínio é apresentada na Figura 3. Pode-se notar por meio desta figura uma tendência ao aumento do preço do alumínio ao longo dos anos.

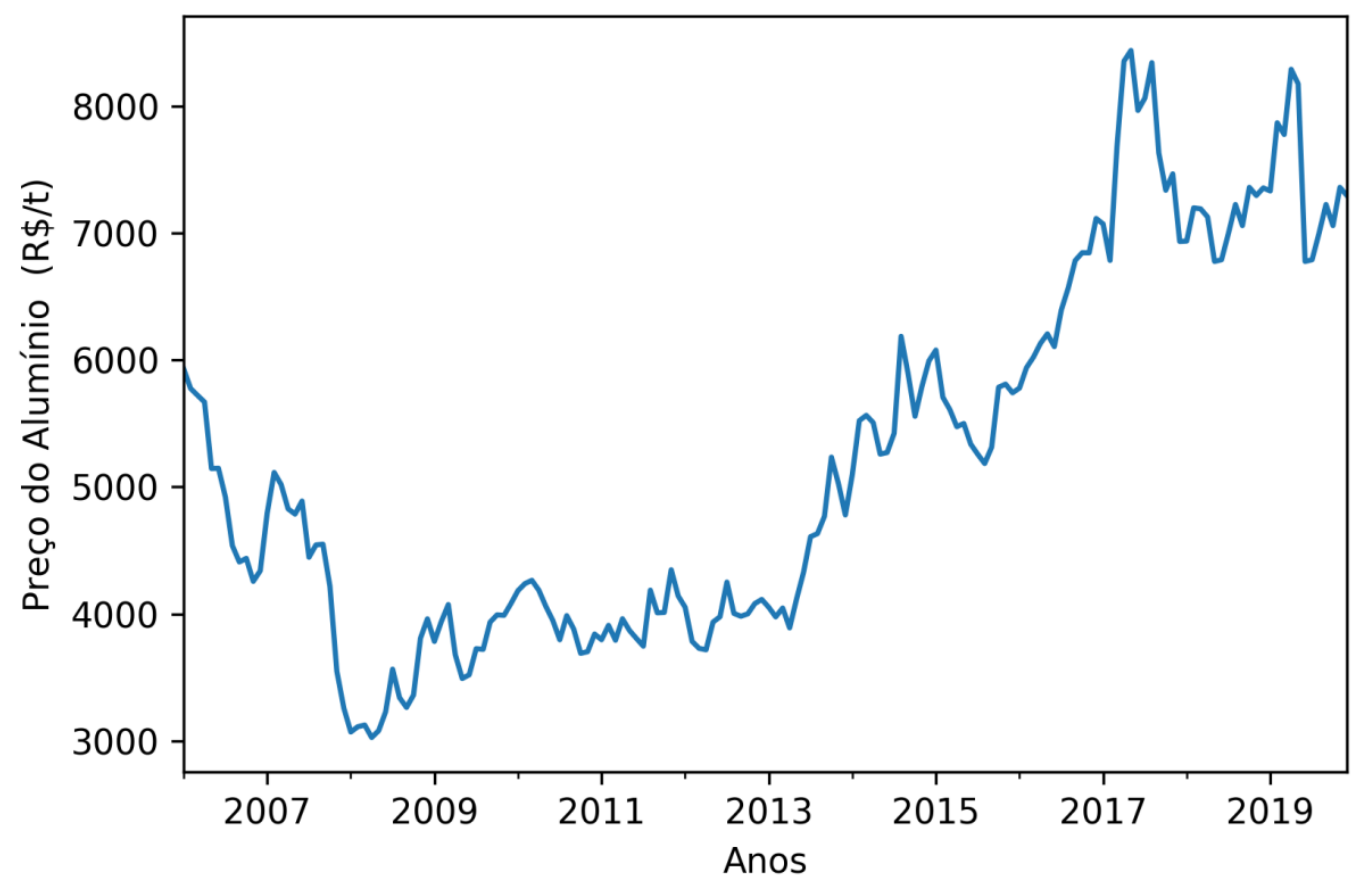

Figura 3. Série: Preço do alumínio

Fonte: Dados da pesquisa (2020).

O preço médio mensal do alumínio, durante o período estudado, é apresentado na Figura 4. Pode-se notar por meio desta figura que o preço médio manteve-se praticamente constante. Apresentando uma pequena queda durante os meses de abril, 
maio, junho e julho. Observou-se um preço médio mínimo de $5015,4 \mathrm{R} \$ / \mathrm{t}$ no mês de maio e um preço médio máximo de $5182,5 \mathrm{R} \$ / \mathrm{kg}$ para o mês de novembro.

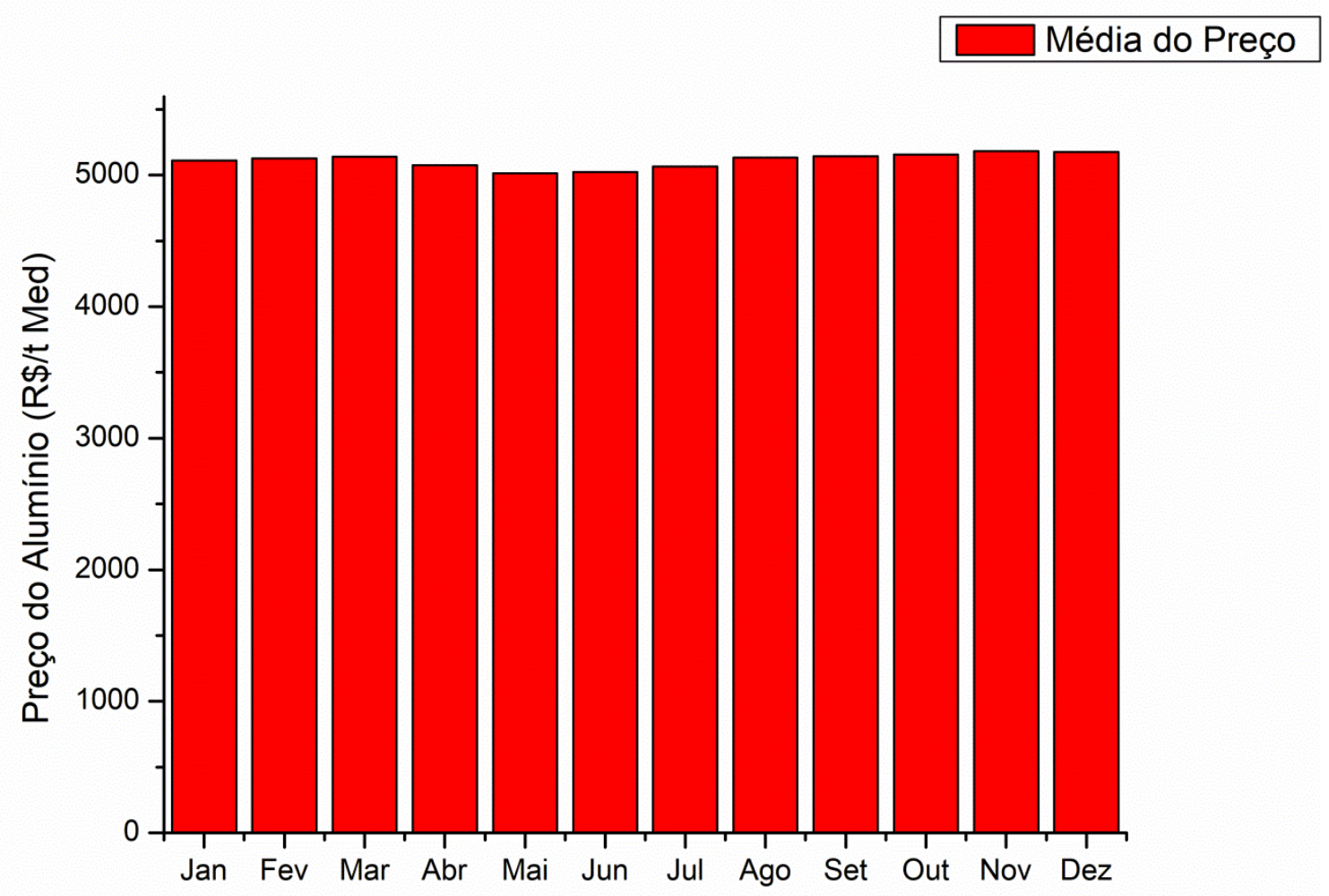

Figura 4. Preço médio mensal do alumínio

Fonte: Dados da pesquisa (2020).

\subsection{Modelos MLP E LSTM}

Neste trabalho implementaram-se modelos de redes neurais MLP e LSTM por meio da biblioteca Keras, rodando como frontend em TensorFlow. Os modelos otimizados, por meio de ajustes nos hiperparâmetros, utilizaram o algoritmo de otimização Adam com os seguintes hiperparâmetros: MLP neurons/LSTM cells $=16$, 
batch $=1$, learning rate $=0.001$ e activate $=$ relu. $\mathrm{Na}$ Tabela 3 , apresentam-se os resultados obtidos dos modelos.

\section{Tabela 3}

Resultados das métricas - conjuntos de treino e teste

\begin{tabular}{l|c|c|c|c|cc}
\hline \multirow{2}{*}{ Cenário } & \multicolumn{3}{|c}{ Treino } & \multicolumn{3}{c}{ Teste } \\
\cline { 2 - 7 } & $\mathbf{r}^{2}$ & RMSE & MAE & $\mathbf{r}^{2}$ & RMSE & MAE \\
\hline LSTM & 0,92 & 228,8 & 172,5 & 0,75 & 410,72 & 277,5 \\
\hline MLP & 0,92 & 219,3 & 165,1 & 0,52 & 570,0 & 475,0 \\
\hline
\end{tabular}

Nota. Fonte: Dados da pesquisa (2020).

Pode-se observar, dos dados apresentados na Tabela 3, que os indicadores $r^{2}$, RMSE e MAE obtidos pelos modelos apresentam valores similares para o conjunto de treino. Os resultados para o conjunto de teste mostram que a rede neural LSTM teve um desempenho melhor.

Na Figura 5, apresenta-se a dispersão dos valores preditos pelos dois algoritmos em relação aos valores observados para a variável de resposta. 

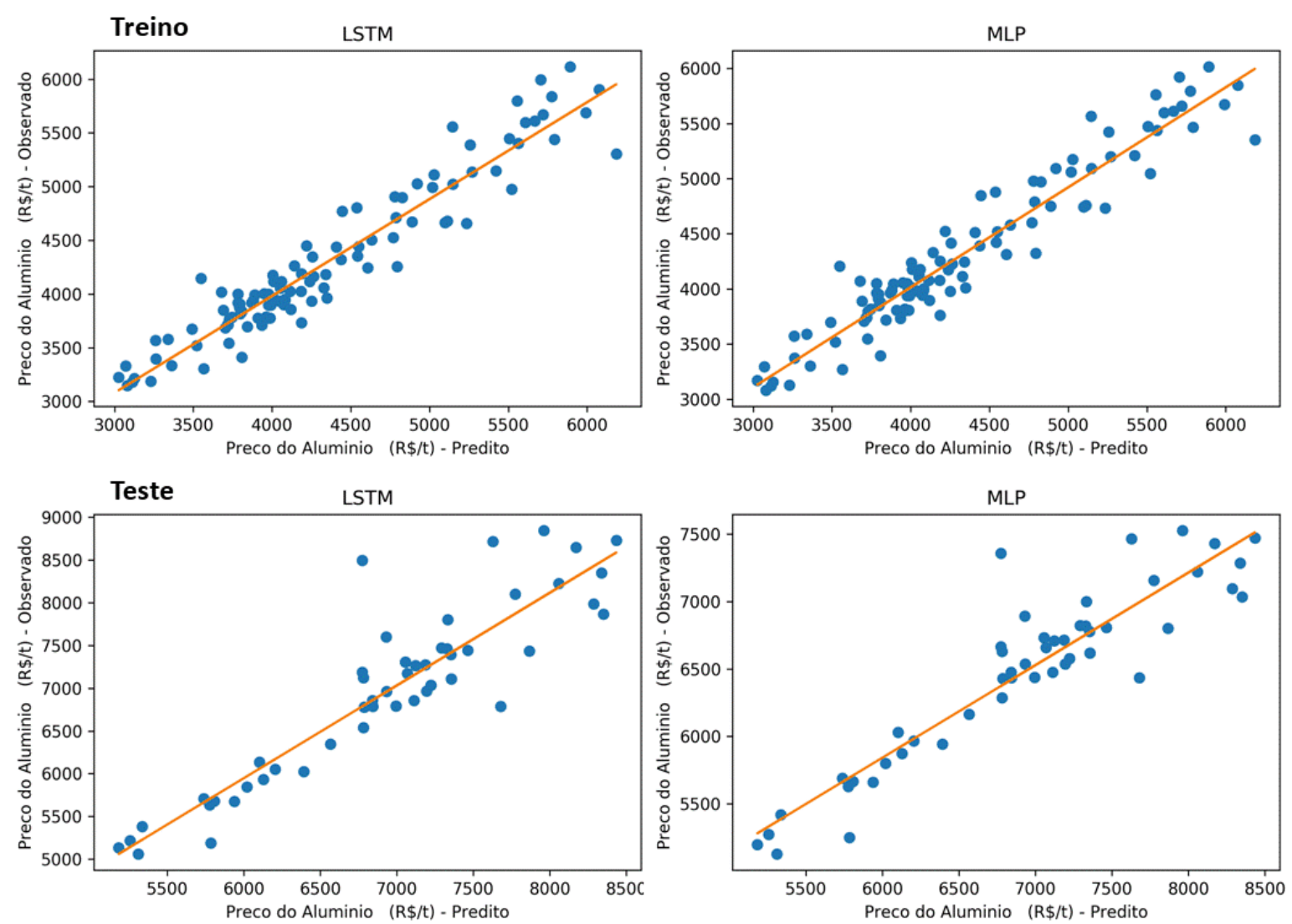

Figura 5. Dados preditos em função de dados observados

Fonte: Dados da pesquisa (2020).

Nas Figuras 6 e 7, apresentam-se os resultados de predição, treinamento e teste (168 meses) para os dois modelos. Pode-se notar, por meio das figuras, que o modelo LSTM para o conjunto de teste obteve melhor aderência dos dados previstos com os dados reais. 


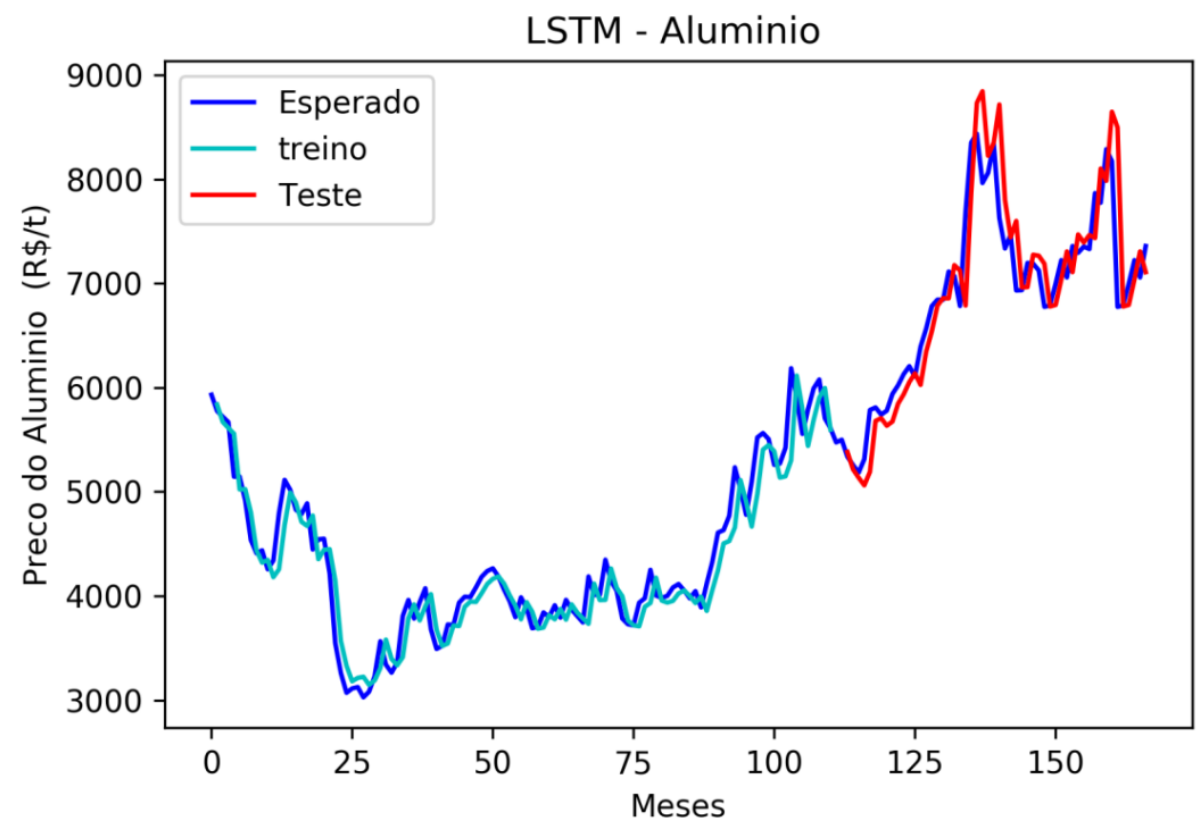

Figura 6. Previsão de treino e teste - LSTM

Fonte: Dados da pesquisa (2020).

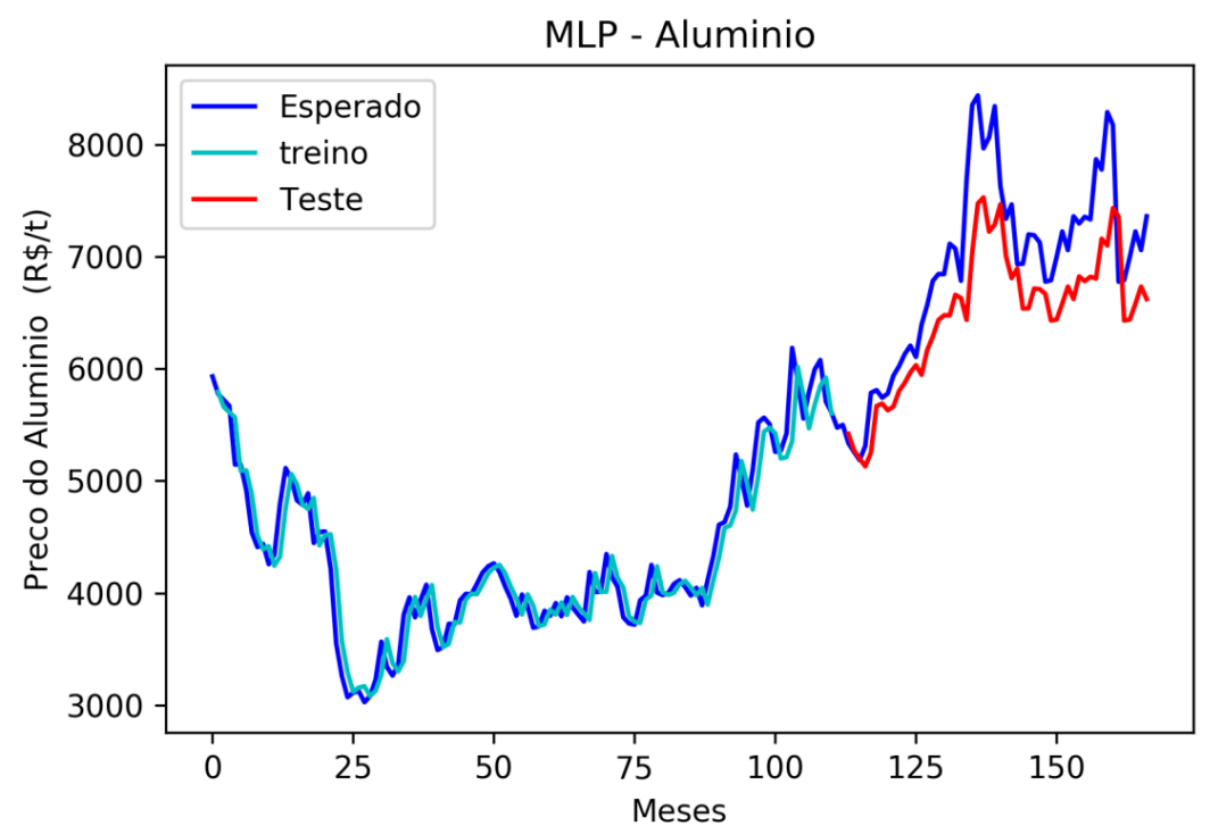

Figura 7. Previsão de treino e teste - MLP

Fonte: Dados da pesquisa (2020). 
Na sequência, realizaram-se previsões do preço do alumínio para os meses de janeiro, fevereiro, março, abril, maio e junho de 2020 que não participaram da etapa de treinamento e teste (Tabela 4). Nesta tabela também são apresentados os Erros Relativos Percentuais (ERP). O ERP é obtido por meio da equação:

$$
E R P=\left|\frac{\text { Observado }- \text { Predito }}{\text { Observado }}\right| \times 100
$$

Tabela 4

Resultados das previsões ( $\mathbf{R} / \mathbf{t})$ e Erros percentuais relativos (\%)

\begin{tabular}{lcccrr}
\hline Mês & WorldBank & LSTM & MLP & ERP_LSTM & \multicolumn{1}{c}{ ERP_MLP } \\
\hline Jan & 7353,49 & 7391,84 & 6765,64 & 0,521521 & 7,994163 \\
Fev & 7329,56 & 7510,51 & 6468,97 & 2,46877 & 11,74136 \\
Mar & 7866,33 & 7655,89 & 6298,29 & 2,675199 & 19,93356 \\
Abr & 7774,11 & 7836,12 & 6198,67 & 0,797648 & 20,26521 \\
Mai & 8287,34 & 8062,88 & 6129,98 & 2,708469 & 26,032 \\
Jun & 8173 & 8353,44 & 6070,78 & 2,207757 & 25,72152 \\
\hline & & Média & 1,896561 & 18,61464 \\
\hline
\end{tabular}

Nota. Fonte: Dados da pesquisa (2020).

Os resultados das previsões, em termos gráficos, são apresentados na Figura 8. 


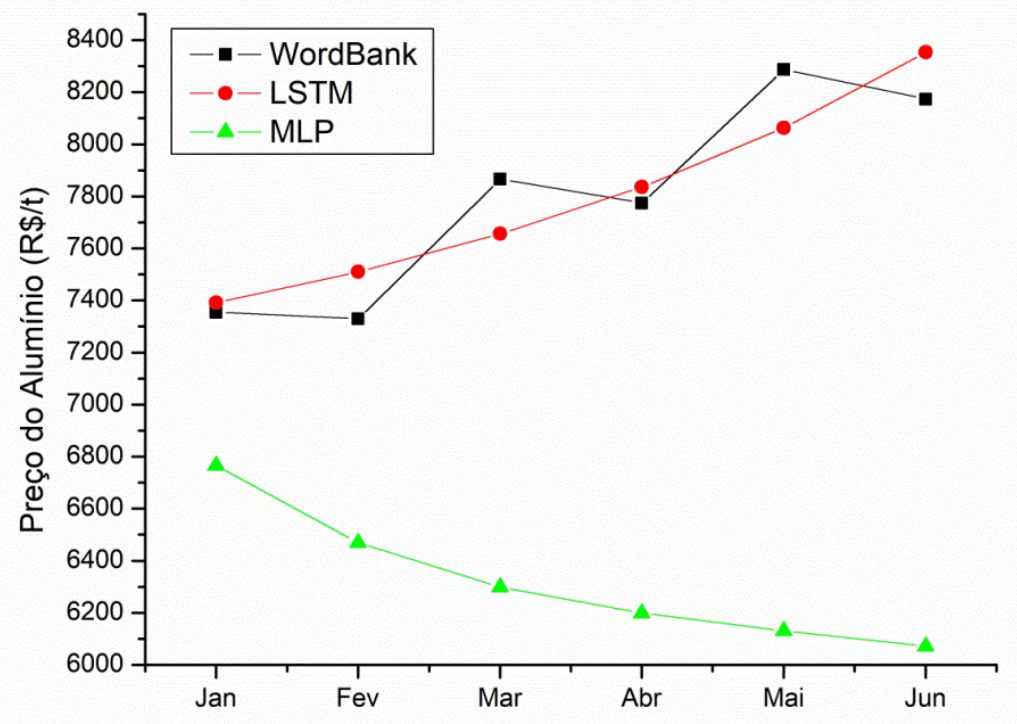

Figura 8. Previsões do preço do alumínio para Jan/Fev/Mar/Abr/Mai/Jun de 2020 (MLP e LSTM)

Fonte: Dados da pesquisa (2020).

Por meio dos resultados apresentados na Tabela 4 e na Figura 8, conclui-se que as previsões do modelo LSTM estão muito mais próximas dos valores fornecidos pelo Word Bank que as previsões do modelo MLP. Observa-se, também, que o modelo LSTM apresenta um erro percentual relativo médio bem menor que o modelo MLP $\left(E R P \_L S T M=1,89 \%\right.$ e ERP_MLP $\left.=18,61 \%\right)$.

\section{CONSIDERAÇÕES FINAIS}

Neste trabalho apresentou-se uma aplicação de modelos de redes neurais artificiais para previsão do preço do alumínio. A série de preços do alumínio, no período entre 2006 e 2020, foi obtida do site do Word Bank. Os modelos passaram pelas fases de preparação de dados, definição das estruturas, estimativas, avaliação dos resultados e validação. 
Observou-se, no processo de treinamento e teste das redes neurais, que o modelo LSTM apresentou para o conjunto de teste um melhor desempenho.

$\mathrm{Na}$ sequência, ressaltou-se para os meses que não participaram do treinamento da rede (janeiro, fevereiro, março, abril, maio e junho de 2020), que as previsões do modelo LSTM foram para a série estudada mais precisas que a do modelo MLP. A proximidade dos valores preditos e reais demonstram, para um horizonte de seis meses, a boa capacidade de generalização da rede LSTM.

Resultados encontrados na literatura especializada e obtidos neste artigo apontam para a eficiência da rede neural recorrente LSTM em previsões de séries temporais.

Apesar do modelo LSTM apresentar resultados adequados para uma previsão de seis meses, sugere-se para outros trabalhos de pesquisa proceder a estimação do preço do alumínio por meio do modelo GRU (Gated Recurrent Unit).

\section{REFERÊNCIAS}

Bastiani, M., Santos, J. A. A., Schmidt, C. A P., \& Sepulveda, G. P. L. (2018). Application of data mining algorithms in the management of the broiler production. Geintec, 8(3), 4573-4587.

Tubino, D. F. (2009). Planejamento e controle da produção-Teoria e Prática. São Paulo: Atlas.

Cankurt, S., \& Subasi, A. (2015). Comparasion of linear regression and neural network models forecasting tourist arrivals to turkey. International Symposium on Sustainable Development, Turquia.

Cardoso, J. R. (2011). A indústria do alumínio: estrutura e tendências. Rio de Janeiro: BNDES.

Castro, J. B. B. (2015). Projeção de preços de alumínio: modelo ótimo por meio de combinações de previsões. (Tese de Doutorado). Universidade de São Paulo, USP, São Paulo. 
Castro, J. B. C., Araujo, A. C., \& Montini, A. A. (2013). Comparative modeling assessment aluminum price forecast. In: XVI Semead. São Paulo: USP.

Graves A. (2014). Towards end-to-end speech recognition with recurrent neural networks. Proceedings of the 31st International Conference on Machine Learning (ICML-14), Beijing, China.

Haykin, S. (2001). Redes neurais: princípios e práticas. Porto Alegre: Bookman.

IndexMundi.(2020). Alumínio preço mensal - Real brasileiro por tonelada. Recuperado de: https://www.indexmundi.com/pt/precos-de mercado/?mercadoria=alumínio\&meses=180\&moeda=brl. . Acess 0 em: $28 / \mathrm{Jul} . / \mathrm{de}$ 2020.

Kriechbaumer, T., Angus, A., Parsons, D., \& Casado, M. R. (2014). An improved wavelet-ARIMA approach for forecasting metal prices. Resources Policy, 39(1), 3241.

Moore, D. S., Mccabe, G. P., Duckworth, W. M., \& Sclove, S. L. A. (2006). A prática da estatística experimental: como usar dados para tomar decisões. Rio de Janeiro: LTC.

Morettin, P. A., \& Toloi, C. M. C. (2004). Análise de séries de temporais. São Paulo: Edgard Blucher.

Nelson, M. Q., Pereira, A C. M., \& Oliveira, A R. A. (2017). Stock market's price prediction with LSTM neural networks. International Joint Conference of Neural Networks (IJCNN), Anchorage, Alaska.

Pinheiro, T. C., Santos, J. A. A., \& Pasa, L. A. (2020). Gestão da produção de frangos de corte por meio de redes neurais artificiais. Revista Holos, 3692), 1-15.

$\mathrm{Ru}, \mathrm{Y} .$, \& Ren, H. (2012). Application of ARMA model in forecasting aluminum price. Applied Mechanics and Materials, 66-71.

Sebastian, S. (2016). Performance evaluation by artificial neural network using WEKA. International Research Journal of Engineering and Technology, 3(1), 1459-1464.

Silva, M. P. (2010). Aplicação de redes neurais artificiais no diagnóstico de falha de turbina a gás. (Tese de Doutorado). Pontifícia Universidade Católica do Rio de Janeiro, PUCRJ, Rio de Janeiro. 
Spancerski, J. A., \& Santos, J. A. A. (2021). Aplicação de redes neurais recorrentes na previsão de geração eólica. Revista Cereus, 13(1), 217-227.

Zao, Z. (2017). LSTM network: a deep learning approach for short-term traffic forecast. IET Intelligent Transport Systems, 11(1), 68-75.

Data de Submissão: 24/11/2020

Data de Aceite: 24/08/2021 